\title{
Dietary patterns and breast cancer risk among women
}

\author{
Zeinab Karimi ${ }^{1}$, Mahsa Jessri ${ }^{2}$, Anahita Houshiar-Rad ${ }^{3}$, Hamid-Reza Mirzaei ${ }^{4}$ and \\ Bahram Rashidkhani ${ }^{1, *}$ \\ 'Department of Community Nutrition, Faculty of Nutrition Sciences and Food Technology, National Nutrition \\ and Food Technology Research Institute (WHO Collaborating Center), Shahid Beheshti University of Medical \\ Sciences and Health Services, 46 West Arghavan St., Farahzadi Blvd, Shahrak Qods, 1981619573 Tehran, \\ Islamic Republic of Iran: ${ }^{2}$ Department of Nutritional Sciences, Faculty of Medicine, University of Toronto, Toronto, \\ ON, Canada: ${ }^{3}$ National Nutrition and Food Technology Research Institute (WHO Collaborating Center), Shahid \\ Beheshti University of Medical Sciences, Tehran, Islamic Republic of Iran: ${ }^{4}$ Department of Radiation Oncology, \\ Faculty of Medicine, Shahid Beheshti University of Medical Sciences, Tehran, Islamic Republic of Iran
}

Submitted 21 October 2011: Final revision received 3 March 2013: Accepted 7 March 2013: First published online 7 May 2013

\begin{abstract}
Objective: Breast cancer is the most common type of cancer in women worldwide. Several studies have examined the role of single nutrients and food groups in breast cancer pathogenesis but fewer investigations have addressed the role of dietary patterns. Our main objective was to identify the relationship between major dietary patterns and breast cancer risk among Iranian women.

Design: Hospital-based case-control study.

Setting: Shohada Teaching Hospital, Tehran, Iran.

Subjects: Overall, 100 female patients aged 30-65 years with breast cancer and 174 female hospital controls were included in the present study. Dietary intake was assessed using a valid and reliable semi-quantitative FFQ consisting of 168 food items.

Results: Two dietary patterns were identified explaining $24 \cdot 31 \%$ of dietary variation in the study population. The 'healthy' food pattern was characterized by the consumption of vegetables, fruits, low-fat dairy products, legumes, olive and vegetable oils, fish, condiments, organ meat, poultry, pickles, soya and whole grains; while the 'unhealthy' food pattern was characterized by the consumption of soft drinks, sugars, tea and coffee, French fries and potato chips, salt, sweets and desserts, hydrogenated fats, nuts, industrial juice, refined grains, and red and processed meat. Compared with the lowest tertile, women in the highest tertile of the 'healthy' dietary pattern score had $75 \%$ decreased risk of breast cancer $(\mathrm{OR}=0 \cdot 25,95 \% \mathrm{CI} 0 \cdot 08,0 \cdot 78)$, whereas women in the highest tertile of the 'unhealthy' dietary pattern had a significantly increased breast cancer risk $(\mathrm{OR}=7 \cdot 78,95 \%$ CI $2 \cdot 31,26 \cdot 22)$.

Conclusions: A healthy dietary pattern may be negatively associated with breast cancer risk, while an unhealthy dietary pattern is likely to increase the risk among Iranian women.
\end{abstract}

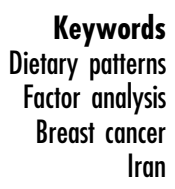

Breast cancer is the most common type of cancer and the leading cause of cancer mortality among women worldwide $^{(1)}$. Although the incidence of female breast cancer in most Asian countries is much lower than that in Western societies ${ }^{(2)}$, the rate has increased steadily in recent years $^{(2-7)}$. In Iran, the age-adjusted incidence rate for breast cancer is 24.42 per 100000 women per year (standardized on the world population).

Although a number of risk factors have been identified for breast cancer, some are difficult to modify (e.g. genetics) while dietary pattern is a modifiable risk factor which could be targeted to prevent and treat breast cancer ${ }^{(2,8,9)}$. Although several studies have examined the role of single micro- nutrients, macronutrients and food items in the pathogenesis of breast cancer, most of the relationship remains controversial as the evidence from these studies does not take into account the complexity of the dietary pattern as a whole ${ }^{(1,6)}$. Diet is a complex combination of nutrients with synergic interactions which should be considered when analysing diet-disease relationships ${ }^{(10)}$. During the past decade interest has shifted to dietary pattern analysis, as it can accommodate the complex interplay of nutrients within a diet and identify patterns in dietary behaviours by representing a broad picture of food and nutrient intakes ${ }^{(4,9,11,12)}$. In addition, dietary patterns form intuitively practical tools for determining public health recommendations ${ }^{(1,4,6,8,10)}$. 
Dietary patterns have previously been assessed in relation to breast cancer risk in several studies conducted in the $\mathrm{USA}^{(7,8,10,11,13,14)}$, Europe ${ }^{(4,6,9,12,15,16)}$, Uruguay ${ }^{(17,18)}$, $\operatorname{Japan}^{(2)}$ and China ${ }^{(3,5)}$, and a reduced risk has been reported among those following a 'prudent' dietary pattern ${ }^{(2,13,17)}$, a 'Mediterranean' pattern ${ }^{(14)}$, a 'traditional Southern' dietary pattern ${ }^{(10)}$, 'stew' and 'traditional' dietary patterns ${ }^{(18)}$, a 'salad vegetable' pattern ${ }^{(12,16)}$, a 'vegetable-soy' pattern ${ }^{(11)}$ and a 'vegetables-fruit-soy-milk-poultry-fish' dietary pattern $^{(3)}$. Compared with the typical 'Western' diet, all of these dietary patterns were higher in fruits and vegetables and lower in animal fats. In contrast, an increased risk of breast cancer has been associated with a 'Western' dietary pattern $^{(14,17,18)}$, a 'starch rich' pattern ${ }^{(15)}$, an 'ethnic-meat/ starch' pattern $^{(11)}$, a 'refined grain-meat-pickle' pattern ${ }^{(1)}$, a 'meat-sweet' pattern ${ }^{(5)}$, a 'Western-alcohol' pattern ${ }^{(4)}$ and a 'drinker' pattern characterized by high intakes of alcoholic beverages ${ }^{(17)}$. Furthermore, adherence to a 'prudent' dietary pattern ${ }^{(14)}$ and an 'animal products' pattern $^{(15)}$ has been positively associated with breast cancer risk, while a 'pork, processed meat and potato' pattern has shown an inverse association ${ }^{(6)}$.

To our knowledge, no previous studies have evaluated the association between dietary patterns and breast cancer risk among Middle-Eastern women. However, dietary patterns vary according to geographic region, socioeconomic status, cultural practices and food preferences and availability ${ }^{(3)}$. Specifically, dietary intake of the MiddleEastern population has its own unique features, being characterized by high intakes of refined grains (white rice and bread) and hydrogenated fats and a greater percentage of energy from carbohydrates ${ }^{(19)}$. With these features, factor analysis may result in different dietary patterns in this region compared with other parts of the world. The aims of the present hospital-based case-control study were therefore to identify major dietary patterns among Iranian women and to examine the relationship between these dietary patterns and breast cancer risk.

\section{Materials and methods}

The present case-control study included 100 female cases aged 30-65 years who were admitted to the major general hospitals in Tehran province, Iran. Cases were diagnosed with incident, histologically confirmed breast cancer within the past 5 months, and they did not have a history of cancers at other sites or hormone replacement therapy. Controls ( $n$ 184) were selected from female patients admitted to the same hospital as the cases for a variety of acute, non-neoplastic conditions unrelated to long-term modification of diet. Controls and cases were frequencymatched by age (5-year groups). Nine controls withdrew during the research and one was excluded due to having incomplete dietary records. Moreover, dietary records with reported energy intakes of $\leq 2100 \mathrm{~kJ} / \mathrm{d}$ or $\geq 21000 \mathrm{~kJ} / \mathrm{d}$ and those with fifty or more skipped food items were considered invalid and were excluded from all analyses. This reduced our sample size to 100 cases and 174 controls (response rate $=96 \cdot 5 \%$ ).

\section{Dietary assessment}

The study data were collected by specifically trained professional interviewers through private face-to-face interviews. Trained dietitians collected the dietary data by means of a validated 168-item semi-quantitative FFQ that was modified to include Iranian food items ${ }^{(20)}$. This FFQ has previously shown relative validity and reproducibility for assessing food and nutrient intakes among Iranian adults and it is an acceptable tool for use in this population $^{(20)}$. Dietary habits of the cases in the year prior to diagnosis and of the controls in the year before the interview were collected. Using this FFQ, the consumption frequency of each food item was evaluated on a daily, weekly or monthly basis, and the portion sizes of consumed foods were specified according the US Department of Agriculture portion sizes (e.g. apple, one medium; bread, one slice; dairy, one cup). Whenever use of the US Department of Agriculture portion sizes was not possible, household measures were used alternatively (e.g. beans, one tablespoon; chicken meat, one leg or wing; rice, one large, medium or small plate).

Intakes of the 168 FFQ food items were reclassified into twenty-six predefined food groups according to the similarity of their nutrient contents (see Appendix) ${ }^{(3,9,16)}$. For each participant, the average daily intake of each food group was calculated by summing the intakes of individual food items within that group.

In addition, the relative accuracy of reported energy intakes was assessed by dividing energy intake by BMR (EI:BMR) in order to control for its confounding effect in the analyses. BMR was calculated according to the Schofield equation using participants' gender, age, weight and height ${ }^{(21)}$. An EI:BMR value of $<1.35$ indicated under-reporting, while a value in the range $1 \cdot 35-2 \cdot 39$ indicated normal reporting and a value $\geq 2 \cdot 40$ indicated over-reporting of energy intake ${ }^{(22)}$.

\section{Other measures}

Weight was measured on a flat, uncarpeted surface using a digital scale (model 803; Seca, Hamburg, Germany) and was recorded to the nearest $0 \cdot 1 \mathrm{~kg}$. Height was measured using a standard stadiometer (model 206 Portable Body Meter Measuring Device; Seca) with the participant's head in the Frankfort horizontal plane, and it was recorded to the nearest $0.5 \mathrm{~cm}$. BMI was calculated by dividing weight by the square of height $\left(\mathrm{kg} / \mathrm{m}^{2}\right)$.

During the face-to-face interviews, physical activity level was assessed using a pre-tested questionnaire and data were expressed as metabolic equivalent hours per day (MET-h/d) ${ }^{(23)}$. Additional information on participants' demographic characteristics, smoking history, alcohol 
consumption, medical history and prescription drug use was also collected using a questionnaire.

\section{Statistical analysis}

Data were analysed using the statistical software package SPSS version $16 \cdot 0$. Factor analysis (principal component analysis) was used to identify major dietary patterns based on the twenty-six predefined food groups (Appendix) and two interpretable factors were retained based on the scree test $^{(24)}$. An orthogonal rotation procedure (varimax rotation) was then applied to simplify the factor structure and render it more easily interpretable. The derived factors were labelled based on their interpretability and review of the literature. The factor score for each pattern was calculated by summing intakes of food groups weighted by factor loadings, and each participant was then assigned a score for each of the identified patterns. A factor loading of $>0 \cdot 3$ was used as a cut-off point to identify the primary factors on which the items were loaded. For further analyses, factor scores were categorized into tertiles of the control group.

To compare general characteristics across the tertile categories of dietary pattern scores, ANOVA and $\chi^{2}$ tests were used as appropriate. The relationship between major dietary patterns and breast cancer risk was assessed using logistic regression analysis in different models, controlling for age (continuous) and menopausal status (postmenopause/premenopause) in model I and for age (continuous), menopausal status (postmenopause/ premenopause), age at menarche (continuous), age at first full-term pregnancy (FFTP; continuous), smoking status (yes/no), oral contraceptive drug use (yes/no), BMI (continuous), relative accuracy of energy reporting (under-reporting/accurate reporting/over-reporting of energy intake), physical activity (continuous) and family history of breast cancer in a first- or second-degree relative (yes/no) in model II. Results are presented as odds ratios and $95 \%$ confidence intervals. Generally, due to the small sample size and for maintaining the statistical power, all individuals with missing answers to any of the confounders were included in a separate 'missing' category (missing indicator method) ${ }^{(25)}$. Thus the same number of women was included in all models, making the comparison possible.

As a basis for trend analyses, ordinal scores were constructed from the categorized variables and were placed into the model as successive integers. All statistical tests were two-sided and $\alpha$ level was set at $P<0 \cdot 05$.

\section{Etbical approval}

The study was conducted based on the Declaration of Helsinki guidelines and all procedures were approved by the ethics board of the Faculty of Nutrition Sciences and Food Technology, National Nutrition and Food Technology Research Institute (WHO Collaborating Center), Shahid Beheshti University of Medical Sciences, Tehran, Iran. Written informed consent was obtained from all participants before data collection began.

\section{Results}

As presented in Table 1, two major dietary patterns were extracted using factor analysis: a 'healthy' dietary pattern (high consumption of vegetables, fruits, low-fat dairy products, legumes, olive and vegetable oils, fish, condiments, organ meat, poultry, pickles, soya and whole grains) and an 'unhealthy' dietary pattern (high in soft drinks, sugars, tea and coffee, French fries and potato chips, salt, sweets and desserts, hydrogenated fats, nuts, industrial juice, refined grains, and red and processed meat). Overall, these factors explained $24 \cdot 31 \%$ of the entire variance.

Selected characteristics of participants within tertiles of dietary pattern scores are shown in Table 2. In comparison with participants in the lowest tertile of the 'healthy' dietary pattern score, those in the highest were significantly younger at FFTP $(P=0 \cdot 03)$, had higher energy intake $(P<0 \cdot 001)$, were more physically active $(P<0 \cdot 01)$, were less likely to have used oral contraceptive drugs $(P<0 \cdot 01)$ and had higher over-reporting of energy intake $(P<0 \cdot 001)$. In contrast, compared with women in the lowest tertile of the 'unhealthy' dietary pattern, those in the highest tertile were less likely to be postmenopausal $(P<0 \cdot 001)$, had higher energy intake $(P<0 \cdot 001)$, were more likely to have used oral contraceptive drugs $(P=0 \cdot 03)$ and had higher over-reporting of energy intake $(P<0 \cdot 001)$. Generally, about $1 \%$ of women consumed alcoholic beverages and $45 \cdot 2 \%$ of women used supplements in this population.

Table 1 Factor-loading matrix for the two major dietary patterns

\begin{tabular}{|c|c|c|}
\hline Food group & $\begin{array}{l}\text { 'Healthy' } \\
\text { dietary } \\
\text { pattern }\end{array}$ & $\begin{array}{c}\text { 'Unhealthy' } \\
\text { dietary } \\
\text { pattern }\end{array}$ \\
\hline Vegetables & 0.727 & -0.102 \\
\hline Fruits & 0.651 & 0.094 \\
\hline Low-fat dairy products & 0.613 & -0.157 \\
\hline Legumes & 0.525 & 0.284 \\
\hline Olive and vegetable oils & 0.472 & 0.062 \\
\hline Condiments & 0.441 & $0 \cdot 166$ \\
\hline Fish & $0 \cdot 440$ & -0.044 \\
\hline Organ meat & 0.407 & 0.089 \\
\hline Poultry & 0.389 & 0.078 \\
\hline Pickles & 0.349 & 0.087 \\
\hline Soya & $0 \cdot 316$ & $-0 \cdot 118$ \\
\hline Whole grains & $0 \cdot 316$ & $0 \cdot 291$ \\
\hline High-fat dairy products & 0.302 & $0 \cdot 180$ \\
\hline Soft drinks & $0 \cdot 127$ & 0.606 \\
\hline Sugars & 0.039 & 0.602 \\
\hline Tea and coffee & 0.054 & 0.576 \\
\hline French fries and potato chips & 0.046 & 0.516 \\
\hline Salt & $-0 \cdot 258$ & 0.448 \\
\hline Sweets and desserts & 0.094 & 0.431 \\
\hline Hydrogenated fats & $0 \cdot 101$ & 0.419 \\
\hline Nuts & 0.351 & 0.412 \\
\hline Industrial juice & 0.086 & 0.402 \\
\hline Refined grains & $-0 \cdot 168$ & 0.385 \\
\hline Red meat & $0 \cdot 276$ & 0.335 \\
\hline Processed meat & $-0 \cdot 207$ & 0.313 \\
\hline Egg & $0 \cdot 119$ & $0 \cdot 294$ \\
\hline Percentage of variance explained & $14 \cdot 67$ & $9 \cdot 64$ \\
\hline
\end{tabular}


Table 2 Participants' characteristics in tertiles of dietary pattern scores: 100 patients aged 30-65 years with breast cancer (cases) and 174 hospital controls, Tehran, Iran

\begin{tabular}{|c|c|c|c|c|c|c|c|c|c|c|c|c|c|c|}
\hline \multirow[b]{3}{*}{ Characteristic } & \multicolumn{7}{|c|}{ Tertile of 'healthy' dietary pattern score } & \multicolumn{7}{|c|}{ Tertile of 'unhealthy' dietary pattern score } \\
\hline & \multicolumn{2}{|c|}{$\mathrm{T} 1$} & \multicolumn{2}{|c|}{$\mathrm{T} 2$} & \multicolumn{2}{|c|}{ T3 } & \multirow[b]{2}{*}{$P^{\star}$} & \multicolumn{2}{|c|}{ T1 } & \multicolumn{2}{|c|}{ T2 } & \multicolumn{2}{|c|}{ T3 } & \multirow[b]{2}{*}{$P^{*}$} \\
\hline & Mean & SD & Mean & SD & Mean & SD & & Mean & SD & Mean & SD & Mean & SD & \\
\hline Weight (kg) & $73 \cdot 3$ & $10 \cdot 8$ & $71 \cdot 2$ & $12 \cdot 4$ & $73 \cdot 5$ & $12 \cdot 1$ & 0.43 & $69 \cdot 9$ & $11 \cdot 8$ & $73 \cdot 1$ & $12 \cdot 1$ & $73 \cdot 4$ & $12 \cdot 0$ & 0.22 \\
\hline Height (cm) & $157 \cdot 8$ & $5 \cdot 2$ & $156 \cdot 8$ & $6 \cdot 2$ & $157 \cdot 3$ & $6 \cdot 8$ & $0 \cdot 71$ & $155 \cdot 5$ & $6 \cdot 9$ & $157 \cdot 9$ & $5 \cdot 9$ & $157 \cdot 7$ & $5 \cdot 8$ & $0 \cdot 10$ \\
\hline Age at menarche (years) & $13 \cdot 8$ & $1 \cdot 5$ & $13 \cdot 6$ & $1 \cdot 7$ & $13 \cdot 7$ & $1 \cdot 6$ & 0.51 & $13 \cdot 5$ & $1 \cdot 6$ & $13 \cdot 6$ & $1 \cdot 4$ & $13 \cdot 9$ & $1 \cdot 7$ & 0.24 \\
\hline Age at FFTP (years) & $21 \cdot 1$ & 4.9 & $21 \cdot 0$ & $4 \cdot 6$ & $19 \cdot 2$ & $3 \cdot 8$ & 0.03 & $19 \cdot 8$ & $4 \cdot 0$ & $21 \cdot 4$ & $5 \cdot 7$ & $20 \cdot 2$ & $3 \cdot 7$ & $0 \cdot 43$ \\
\hline BMl $\left(\mathrm{kg} / \mathrm{m}^{2}\right)$ & $29 \cdot 1$ & $4 \cdot 2$ & $29 \cdot 1$ & $4 \cdot 4$ & $30 \cdot 0$ & $3 \cdot 7$ & 0.37 & $28 \cdot 9$ & $4 \cdot 1$ & $29 \cdot 5$ & $4 \cdot 7$ & $29 \cdot 7$ & $3 \cdot 8$ & $0 \cdot 60$ \\
\hline Physical activity level (MET-h/d) & $37 \cdot 5$ & $3 \cdot 7$ & $38 \cdot 7$ & $4 \cdot 1$ & $39 \cdot 8$ & $5 \cdot 3$ & $<0.01$ & $38 \cdot 9$ & $4 \cdot 1$ & $38 \cdot 7$ & $3 \cdot 8$ & $38 \cdot 4$ & $5 \cdot 0$ & 0.76 \\
\hline \multirow[t]{2}{*}{ Total energy intake $(\mathrm{kJ} / \mathrm{d})$} & 8556 & 3201 & 11054 & 2723 & 14769 & 4049 & $<0.001$ & 8975 & 3047 & 9591 & 2891 & 13909 & 3975 & $<0.001$ \\
\hline & $n$ & $\%$ & $n$ & $\%$ & $n$ & $\%$ & & $n$ & $\%$ & $n$ & $\%$ & $n$ & $\%$ & \\
\hline \multicolumn{15}{|l|}{ Menopausal status } \\
\hline Premenopause & 67 & 72 & 61 & 59 & 44 & 57 & $0 \cdot 8$ & 28 & 41 & 62 & 69 & 82 & 70 & $<0.001$ \\
\hline Postmenopause & 26 & 28 & 42 & 40 & 33 & 42 & & 40 & 58 & 27 & 30 & 34 & 29 & \\
\hline \multicolumn{15}{|l|}{ Alcohol intake } \\
\hline No & 87 & 98 & 98 & 99 & 73 & 98 & $1 \cdot 00$ & 65 & 100 & 83 & 98 & 110 & 98 & 0.79 \\
\hline Yes & 1 & 1 & 1 & 1 & 1 & 1 & & 0 & 0 & 1 & 1 & 2 & 1 & \\
\hline \multicolumn{15}{|l|}{ Smoking status } \\
\hline No & 90 & 98 & 94 & 94 & 73 & 97 & 0.36 & 65 & 98 & 84 & 98 & 108 & 94 & 0.32 \\
\hline Yes & 2 & 2 & 6 & 6 & 2 & 3 & & 1 & 1 & 2 & 2 & 7 & 6 & \\
\hline \multicolumn{15}{|l|}{ Family history of breast cancert } \\
\hline No & 47 & 72 & 79 & 81 & 63 & 83 & 0.27 & 46 & 79 & 54 & 76 & 89 & 81 & 0.73 \\
\hline Yes & 18 & 28 & 19 & 19 & 13 & 17 & & 12 & 21 & 17 & 24 & 21 & 19 & \\
\hline \multicolumn{15}{|l|}{ Oral contraceptive drug use } \\
\hline No & 70 & 75 & 89 & 86 & 73 & 94 & $<0.01$ & 65 & 94 & 71 & 80 & 96 & 83 & 0.03 \\
\hline Yes & 23 & 25 & 14 & 14 & 5 & 6 & & 4 & 6 & 18 & 20 & 20 & 17 & \\
\hline \multicolumn{15}{|l|}{ Supplement use } \\
\hline No & 50 & 54 & 60 & 59 & 38 & 50 & $0 \cdot 50$ & 39 & 57 & 48 & 55 & 61 & 53 & 0.85 \\
\hline Yes & 42 & 46 & 42 & 41 & 38 & 50 & & 29 & 43 & 39 & 45 & 54 & 47 & \\
\hline \multicolumn{15}{|l|}{ Energy intake reporting } \\
\hline Under-reporting of energy intakeł & 9 & $25 \cdot 7$ & 10 & $12 \cdot 8$ & 1 & $1 \cdot 5$ & $<0.001$ & 14 & $28 \cdot 6$ & 4 & $8 \cdot 2$ & 2 & $2 \cdot 4$ & $<0.001$ \\
\hline Accurate reporting of energy intake $\S$ & 22 & $62 \cdot 9$ & 57 & $73 \cdot 1$ & 32 & $47 \cdot 1$ & & 33 & $67 \cdot 3$ & 42 & $85 \cdot 7$ & 36 & $43 \cdot 4$ & \\
\hline Over-reporting of energy intakell & 4 & $11 \cdot 4$ & 11 & $14 \cdot 1$ & 35 & $51 \cdot 5$ & & 2 & $4 \cdot 1$ & 3 & $6 \cdot 1$ & 45 & $54 \cdot 2$ & \\
\hline
\end{tabular}

T1, first tertile (lowest); T2, second tertile; T3, third tertile (highest); FFTP, first full-term pregnancy; MET, metabolic equivalents; El, energy intake.

*ANOVA for quantitative variables and $\chi^{2}$ test for qualitative variables.

tFamily history of breast cancer in a first- or second-degree relative.

FEl:BMR $<1.35$.

. 
Table 3 Odds ratios (95\% confidence intervals) for breast cancer risk across tertiles of dietary pattern scores*: 100 patients aged $30-65$ years with breast cancer (cases) and 174 hospital controls, Tehran, Iran

\begin{tabular}{|c|c|c|c|c|c|c|}
\hline \multirow[b]{3}{*}{ Dietary pattern } & \multicolumn{5}{|c|}{ Tertile of dietary pattern score } & \multirow[b]{3}{*}{$P$ for trenc } \\
\hline & \multirow[b]{2}{*}{$\mathrm{T} 1$} & \multicolumn{2}{|c|}{$\mathrm{T} 2$} & \multicolumn{2}{|c|}{ T3 } & \\
\hline & & OR & $95 \% \mathrm{Cl}$ & OR & $95 \% \mathrm{Cl}$ & \\
\hline \multirow{2}{*}{\multicolumn{7}{|c|}{$\begin{array}{l}\text { Healthy dietary pattern } \\
\text { Cases/controls }\end{array}$}} \\
\hline & & & & & & \\
\hline$n$ & $35 / 58$ & \multicolumn{2}{|c|}{$46 / 57$} & \multicolumn{2}{|c|}{$19 / 59$} & \\
\hline$\%$ & $35 / 33$ & \multicolumn{2}{|c|}{$46 / 33$} & \multicolumn{2}{|c|}{$19 / 34$} & \\
\hline Model It & 1.00 & $1 \cdot 33$ & $0 \cdot 75,2 \cdot 37$ & 0.53 & $0 \cdot 27,1 \cdot 05$ & $0 \cdot 10$ \\
\hline Model II & $1 \cdot 00$ & 0.45 & $0 \cdot 16,1 \cdot 24$ & $0 \cdot 25$ & $0 \cdot 08,0 \cdot 78$ & 0.02 \\
\hline \multirow{2}{*}{\multicolumn{7}{|c|}{$\begin{array}{l}\text { Unhealthy dietary pattern } \\
\text { Cases/controls }\end{array}$}} \\
\hline & & & & & & \\
\hline$n$ & $11 / 58$ & \multirow{2}{*}{\multicolumn{2}{|c|}{$\begin{array}{l}31 / 58 \\
31 / 33\end{array}$}} & \multirow{2}{*}{\multicolumn{2}{|c|}{$\begin{array}{l}58 / 58 \\
58 / 33\end{array}$}} & \\
\hline$\%$ & $11 / 33$ & & & & & \\
\hline Model It & $1 \cdot 00$ & 3.04 & $1 \cdot 36,6 \cdot 78$ & $5 \cdot 94$ & $2 \cdot 74,12 \cdot 89$ & $<0.001$ \\
\hline Model II & $1 \cdot 00$ & 3.96 & $1 \cdot 10,14 \cdot 25$ & $7 \cdot 78$ & $2 \cdot 31,26 \cdot 22$ & 0.001 \\
\hline
\end{tabular}

Table 3 presents the odds ratio for breast cancer risk across tertiles of dietary pattern scores. After multivariable adjustment for age and menopausal status (model I), no significant association was observed between the healthy' dietary pattern and breast cancer risk ( $P$ for trend $=$ $0 \cdot 10$ ), while the highest tertile of the 'unhealthy' dietary pattern was associated with a 5.94-fold increase in breast cancer risk ( $P$ for trend $<0.001$ ) compared with the lowest tertile. After further adjustment for all confounders in model II (age, menopausal status, age at menarche, age at FFTP, smoking status, oral contraceptive drug use, BMI, relative accuracy of energy reporting, physical activity and family history of breast cancer in a first- or second-degree relative), women in the highest tertile of the 'healthy' dietary pattern had $75 \%$ decreased risk of breast cancer ( $P$ for trend $=0.02$ ) while those in the highest tertile of the 'unhealthy' pattern had $7 \cdot 78$-fold increased risk of breast cancer ( $P$ for trend $=0 \cdot 001$ ) compared with those in the lowest.

Further effect modification by menopausal status revealed that breast cancer risk was not significantly different between premenopausal and postmenopausal women $(P=0.99)$. Table 4 presents the odds ratio for breast cancer risk across tertiles of dietary pattern scores based on menopausal status. After multivariable adjustment (age, menopausal status, age at menarche, age at FFTP, smoking status, oral contraceptive drug use, BMI, energy intake, physical activity and family history of breast cancer in a first- or second-degree relative), the $P$ value for the interaction term suggested that there was no significant difference in dietary patterns of menopausal and non-menopausal women in relation to risk of breast cancer ( $P$ for interaction ('healthy' dietary pattern) $=0 \cdot 23, \quad P$ for interaction ('unhealthy' dietary pattern) $=0 \cdot 14)$.

\section{Discussion}

Two major dietary patterns were identified in this population of Iranian women that together explained $24 \cdot 31 \%$ of the variance in dietary intakes as measured by the FFQ. These patterns were similar to the healthy and unhealthy dietary patterns found in other studies conducted in Iran which have used the same $\mathrm{FFQ}^{(24,26)}$. After adjusting for potential confounders (age, menopausal status, age at menarche, age at FFTP, smoking status, oral contraceptive drug use, BMI, relative accuracy of energy reporting, physical activity and family history of breast cancer in a first- or second-degree relative), those in the highest tertile of the 'healthy' dietary pattern had $75 \%$ lower risk of breast cancer, while being in the highest tertile of the 'unhealthy' pattern was linked to $7 \cdot 78$-fold increased risk for breast cancer, compared with being in the lowest tertile. The odds for the 'unhealthy' dietary pattern increased from OR $=5 \cdot 94(95 \%$ CI $2 \cdot 74,12 \cdot 89)$ to $\mathrm{OR}=7 \cdot 78$ (95\% CI $2 \cdot 31,26 \cdot 22)$ from model I to model II, respectively, and the $95 \%$ confidence intervals were wide, which may be due to the small sample size and use of a large number of adjusting variables in model II.

Generally, findings from previous studies have been inconclusive. Some case-control studies have found an inverse association between breast cancer risk and a healthy dietary pattern or patterns similar to a healthy pattern $^{(2,3,11,14,17,18)}$ and a positive association has been reported between Western or Western-like dietary patterns and breast cancer risk ${ }^{(5,11,14,15,17,18)}$. Higher scores on the 'vegetable-fruit-soy-milk-poultry-fish' dietary pattern were associated with a decreased breast cancer risk among Chinese women $(\mathrm{OR}=0 \cdot 26,95 \%$ CI $0 \cdot 17$, $0 \cdot 42)^{(3)}$. In addition, being in the highest quartile of a 'prudent' dietary pattern, defined as high consumption of 
Table 4 Odds ratios (95\% confidence intervals) for breast cancer risk across tertile categories of dietary pattern scores by menopausal status*: 100 patients aged 30-65 years with breast cancer (cases) and 174 hospital controls, Tehran, Iran

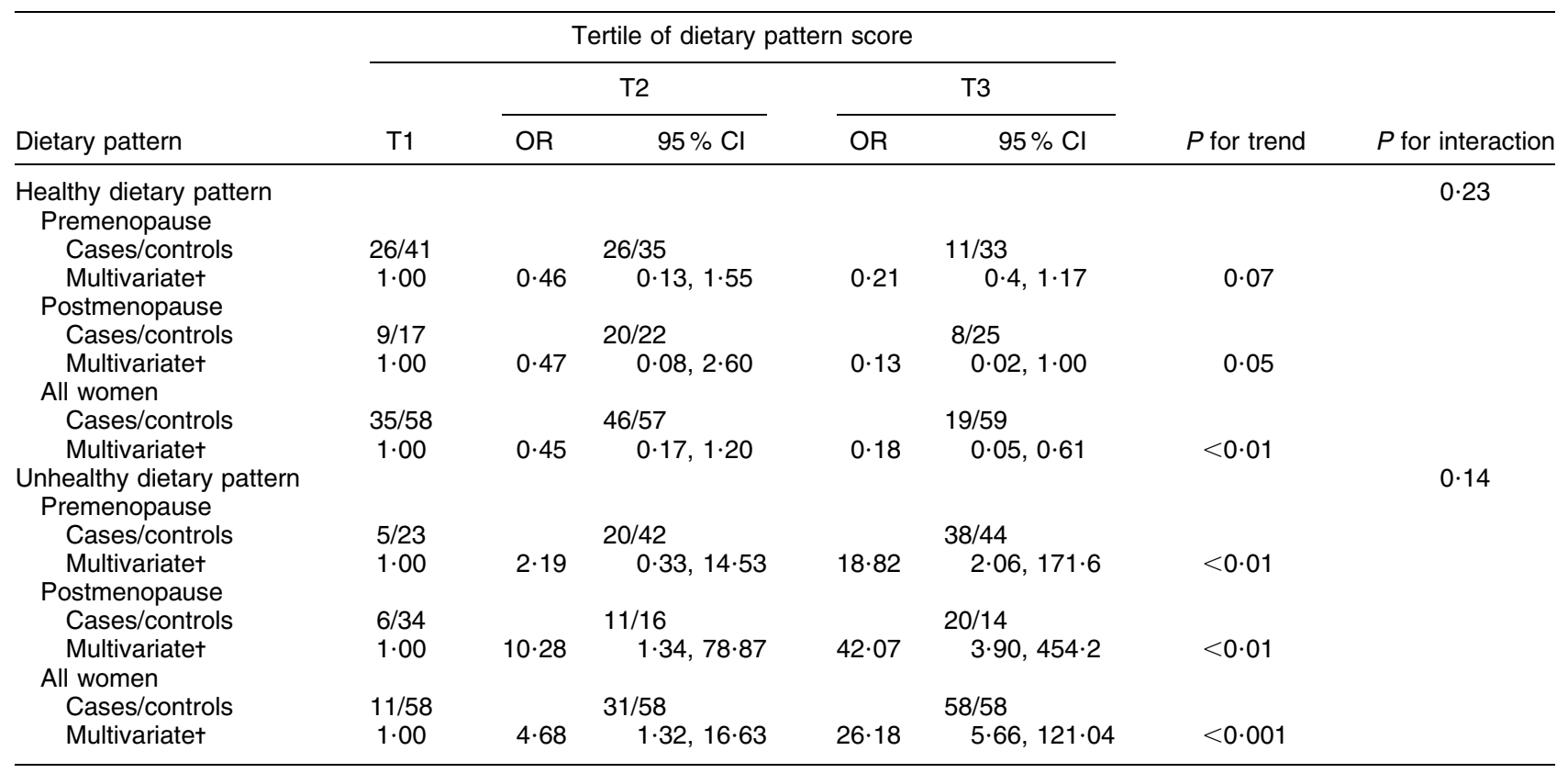

T1, first tertile (lowest); T2, second tertile; T3, third tertile (highest).

${ }^{*}$ All OR were calculated from logistic regression analysis.

tAdjusted for age, age at menarche, age at first full-term pregnancy, smoking status, oral contraceptive drug use, BMI, energy intake, physical activity and family history of breast cancer.

vegetables, fruits and fish, was shown likely to decrease the breast cancer risk by $27 \%$ among Japanese women $(\mathrm{OR}=0.73,95 \% \text { CI } 0.63,0.84)^{(2)}$. Among Uruguay women, a 'healthy' dietary pattern characterized by high loadings of raw vegetables, cooked vegetables, total fruits, poultry and fish was inversely related to breast cancer risk $(\mathrm{OR}=0 \cdot 46,95 \% \mathrm{CI} 0 \cdot 31,0 \cdot 69)^{(18)}$. A prospective cohort study in the USA showed that the 'traditional Southern' pattern characterized by high intakes of cooked greens, beans, legumes, cabbage, sweet potatoes and cornbread may reduce the risk of invasive breast cancer significantly (relative risk $=0 \cdot 78,95 \%$ CI $0 \cdot 65,0 \cdot 95)^{(10)}$. In addition, another cohort study in Australia supported the hypothesis that a dietary pattern rich in fruit and salad might protect against invasive breast cancer and that the effect might be stronger for oestrogen receptor- and progesterone receptor-negative tumours (hazard ratio $=0 \cdot 48$, $95 \%$ CI $0 \cdot 26,0 \cdot 86)^{(27)}$. Similarly, adherence to the 'vegetable-soy' pattern/'prudent' pattern/healthy 'Mediterranean' pattern has been inversely linked with the breast cancer risk in Asian American women ( $P$ for trend $=$ $0 \cdot 013$, case-control study $)^{(11)}$, African American women $(P \text { for trend }=0 \cdot 06 \text {, cohort study })^{(13)}$ and French women $(P \text { for trend }=0 \cdot 003 \text {, cohort study })^{(4)}$.

Overall, a recent meta-analysis of sixteen studies examining the association of dietary patterns and breast cancer risk concluded that being in the highest categories of the 'prudent'/'healthy' dietary patterns reduces the breast cancer risk significantly $(\mathrm{OR}=0 \cdot 89,95 \%$ CI $0 \cdot 82$,
0.99) ${ }^{(28)}$. However, a 'prudent' dietary pattern characterized by high intakes of low-fat dairy products, fruits and vegetables, whole grains, legumes and soups has been associated with an increased breast cancer risk among American women in a case-control study ${ }^{(14)}$. In addition, some case-control ${ }^{(5)}$ and prospective cohort ${ }^{(6,7,16)}$ studies have not found any significant associations between healthy dietary patterns and breast cancer risk. The cancerprotective effects of a healthy dietary pattern may be related, in part, to the higher dietary fibre and antioxidant vitamins included in these patterns. Specifically, it has been suggested that inadequate intakes of green vegetables could result in folic acid deficiency in unhealthy dietary patterns which could reduce the availability of $S$-adenosyl methionine for DNA methylation and thereby influence gene expression $^{(2,4,10,13)}$.

Case-control studies among Chinese women have found that a 'refined grain-meat-pickle' pattern ( $\mathrm{OR}=$ $2 \cdot 58,95 \%$ CI $1.53,4.34)^{(3)}$ and a 'meat-sweet' pattern $(\mathrm{OR}=1 \cdot 3,95 \% \mathrm{CI} 1 \cdot 0,1 \cdot 7)^{(5)}$ could increase the breast cancer risk significantly. In addition, a 'Western' pattern characterized by high consumption of fried, barbecued and processed meat has been positively associated with breast cancer risk $(\mathrm{OR}=2 \cdot 16,95 \%$ CI $1 \cdot 46,3 \cdot 29)$ in Uruguay $^{(18)}$. A study among Italian women has found an increased breast cancer risk among women with a 'starch rich' dietary pattern (high in bread, pasta dishes, cakes and desserts; OR $=1 \cdot 3495 \%$ CI $1 \cdot 10,1 \cdot 65)^{(15)}$. Similarly, a 'Western' dietary pattern characterized by higher intakes 
of processed and red meats, refined grains, sweets and desserts has been associated with increased breast cancer risk among smokers in the USA in a cohort study (relative risk $=1 \cdot 44,95 \% \mathrm{CI} 1 \cdot 02,2 \cdot 03)^{(8)}$. The E3N-EPIC prospective cohort study has also found that a 'Western-alcohol' pattern characterized by meat products, French fries, appetizers, rice/pasta, potatoes and canned fish may be positively associated with breast cancer risk (hazard ratio $=1 \cdot 20,95 \%$ CI $1 \cdot 03,1 \cdot 38)$ among French women ${ }^{(4)}$. Only two studies have found that a 'pork, processed meat and potato' dietary pattern (relative risk $=0.69,95 \%$ CI $0.52,0.92)^{(6)}$ and an 'animal products' pattern $(\mathrm{OR}=0 \cdot 74,95 \% \mathrm{CI} 0 \cdot 61,0 \cdot 91)^{(15)}$ have negative associations with breast cancer risk, while other cohort studies have failed to show any significant relationship $^{(7,10-13,16)}$. Several biological mechanisms may explain the positive association between unhealthy dietary patterns and breast cancer risk. Processed meat is a source of carcinogens such as heterocyclic amines, $\mathrm{N}$-nitroso compounds and polycyclic aromatic hydrocarbons that increase mammary tumours in animal models and are hypothesized to increase breast cancer risk in human subjects $^{(3)}$. In addition, heterocyclic amines and polycyclic aromatic hydrocarbons are not restricted to processed meats; these are largely formed in unprocessed meats by high-temperature cooking.

To our knowledge, the present study is the first one in a Middle-Eastern country to report the association between major dietary patterns and breast cancer risk. The study has several strengths; the first one being the high participation rate, as $100 \%$ of cases and $94.6 \%$ of controls who were initially invited to participate in the research were retained in the final analyses. In addition, using a valid and reliable FFQ for evaluating the dietary intakes increased the data quality ${ }^{(20)}$. One other strength is that the study was conducted in a province with a very high point prevalence of breast cancer where the risk factors for this malignancy are not yet fully known. Findings from the present research could potentially be used in designing interventional strategies targeting dietary intake modifications in order to decrease the breast cancer risk. Studies in developing countries can provide unique opportunities to test the association between dietary patterns and cancer risk ${ }^{(26)}$. Generally, where economic resources are severely restricted, food intake is strongly linked to income, so that even small economic differences are directly reflected in dietary intakes. This linkage would tend to increase the between-person variation. Furthermore, in developing countries, socio-economic backgrounds are quite different from those in the Western world (mainly the status of women, parity, autonomy, work participation, family size and access to the healthcare system), which might influence the relationship between dietary patterns and disease outcomes observed in these countries.

Before the implications of the present study are discussed, it is necessary to consider potential limitations.
First, since dietary intakes were assessed using an FFQ, measurement errors were inevitable, which may have led to underestimation of some associations. However, we compensated for this limitation by using a validated FFQ and excluding over- and under-reporters of energy intake. Second, controls were selected from patients with other diseases (hospital-based case-control design), which is a weakness since their exposure may not be representative of that in members of the study population who are at risk of becoming cases. Third, we cannot entirely rule out the likelihood of residual confounding due to imprecise measurement of important covariates. Being a case-control study, recall bias was also inevitable as patients may recall their diets differently after a cancer diagnosis and there is the possibility that cases who are aware of any possible associations between diet and breast cancer recall exposure more than non-cases. However, we tried to reduce the recall bias through recruiting hospital controls whose medical conditions were unrelated to diet or other major risk factors of breast cancer. In addition, incident cases were registered at a median of 2.5 months after diagnosis and all FFQ were administered by trained dietitians to reduce the recall bias. Moreover, since participants were selected from a population that was readily accessible and convenient through a non-probability sampling strategy, findings may not be generalizable to the entire population. However, recruiting hospital controls using the same convenient sampling strategy is likely to have reduced the impact of non-random sampling. Another limitation of the current study is the presence of missing values for several variables, which may have influenced the results in the multivariate analyses. In addition, our study had mainly the character of a pilot hypothesis-generating study, which allows a further search for breast cancer risk factors. Another limitation stems from the several subjective or arbitrary decisions in the use of factor analysis as the investigator is forced to specify the number of factors. Although eigenvalues, scree plots and interpretability are used to guide the investigator in determining the best factor solution, ultimately such a decision is subjective ${ }^{(24)}$.

Overall, some of the differences in the association of dietary patterns and breast cancer risk reported in previous studies may be related to participants' characteristics. Nevertheless, evaluation of the diet-cancer relationship is complex and requires stratification by multiple characteristics.

\section{Conclusion}

Our findings suggest that a 'healthy' dietary pattern, characterized by high consumption of vegetables, fruits, low-fat dairy products, legumes, olive and vegetable oils, fish, condiments, organ meat, poultry, pickles, soya and whole grains, may protect against risk of breast cancer, while an 'unhealthy' dietary pattern with high consumption 
of soft drinks, sugars, tea and coffee, French fries and potato chips, salt, sweets and desserts, hydrogenated fats, nuts, industrial juice, refined grains, and red and processed meat might be associated with higher risk of breast cancer. However, these findings await replication in largescale longitudinal studies to control for potential biases associated with small sample size and wide confidence intervals in the present research.

\section{Acknowledgements}

Sources of funding: This study was funded by the National Nutrition and Food Technology Research Institute, Shahid Beheshti University of Medical Sciences, Tehran, Iran. Conflicts of interest: The authors have no conflicts of interest. Authors' contributions: Z.K. and B.R. designed the study. A.H. and H.-R.M. helped in data collection. M.J. revised the manuscript, provided input and consultation, and finalized the data and manuscript. Acknowledgements: The authors wish to thank all of the women who participated in the current study.

\section{References}

1. Edefonti V, Randi G, La Vecchia CF et al. (2009) Dietary patterns and breast cancer: a review with focus on methodological issues. Nutr Rev 67, 297-314.

2. Hirose K, Matsuo K, Iwata H et al. (2007) Dietary patterns and the risk of breast cancer in Japanese women. Cancer Sci $98,1431-1438$.

3. Zhang CX, Ho SC, Fu JH et al. (2011) Dietary patterns and breast cancer risk among Chinese women. Cancer Causes Control 22, 115-124.

4. Cottet V, Touvier M, Fournier A et al. (2009) Postmenopausal breast cancer risk and dietary patterns in the E3N-EPIC prospective cohort study. Am J Epidemiol 170, $1257-1267$.

5. Cui X, Dai Q, Tseng M et al. (2007) Dietary patterns and breast cancer risk in the shanghai breast cancer study. Cancer Epidemiol Biomarkers Prev 16, 1443-1448.

6. Mannisto S, Dixon LB, Balder HF et al. (2005) Dietary patterns and breast cancer risk: results from three cohort studies in the DIETSCAN project. Cancer Causes Control 16, 725-733

7. Adebamowo CA, Hu FB, Cho E et al. (2005) Dietary patterns and the risk of breast cancer. Ann Epidemiol 15, 789-795.

8. Fung TT, Hu FB, Holmes MD et al. (2005) Dietary patterns and the risk of postmenopausal breast cancer. Int J Cancer 116, 116-121.

9. Buck K, Vrieling A, Flesch-Janys D et al. (2011) Dietary patterns and the risk of postmenopausal breast cancer in a German case-control study. Cancer Causes Control 22, 273-282.

10. Velie EM, Schairer C, Flood A et al. (2005) Empirically derived dietary patterns and risk of postmenopausal breast cancer in a large prospective cohort study. Am J Clin Nutr 82, 1308-1319.
11. Wu AH, Yu MC, Tseng CC et al. (2009) Dietary patterns and breast cancer risk in Asian American women. Am J Clin Nutr 89, 1145-1154.

12. Sieri S, Krogh V, Pala V et al. (2004) Dietary patterns and risk of breast cancer in the ORDET cohort. Cancer Epidemiol Biomarkers Prev 13, 567-572.

13. Agurs-Collins T, Rosenberg L, Makambi K et al. (2009) Dietary patterns and breast cancer risk in women participating in the Black Women's Health Study. Am J Clin Nutr 90, 621-628.

14. Murtaugh MA, Sweeney C, Giuliano AR et al. (2008) Diet patterns and breast cancer risk in Hispanic and nonHispanic white women: the Four-Corners Breast Cancer Study. Am J Clin Nutr 87, 978-984.

15. Edefonti V, Decarli A, La Vecchia C et al. (2008) Nutrient dietary patterns and the risk of breast and ovarian cancers. Int J Cancer 122, 609-613.

16. Sant M, Allemani C, Sieri S et al. (2007) Salad vegetables dietary pattern protects against HER-2-positive breast cancer: a prospective Italian study. Int J Cancer 121, 911-914.

17. De Stefani E, Deneo-Pellegrini H, Boffetta P et al. (2009) Dietary patterns and risk of cancer: a factor analysis in Uruguay. Int J Cancer 124, 1391-1397.

18. Ronco AL, De Stefani E, Boffetta P et al. (2006) Food patterns and risk of breast cancer: a factor analysis study in Uruguay. Int J Cancer 119, 1672-1678.

19. Esmaillzadeh A \& Azadbakht L (2008) Major dietary patterns in relation to general obesity and central adiposity among Iranian women. J Nutr 138, 358-363.

20. Mirmiran P, Esfahani FH, Mehrabi Y et al. (2010) Reliability and relative validity of an FFQ for nutrients in the Tehran Lipid and Glucose Study. Public Health Nutr 13, 654-662.

21. Goldberg GR, Black AE, Jebb SA et al. (1991) Critical evaluation of energy intake data using fundamental principles of energy physiology: 1. Derivation of cut-off limits to identify under-recording. Eur J Clin Nutr 45, 569-581.

22. Johansson L, Solvoll K, Bjorneboe GE et al. (1998) Underand overreporting of energy intake related to weight status and lifestyle in a nationwide sample. Am J Clin Nutr 68, 266-274.

23. Aaron DJ, Kriska AM, Dearwater SR et al. (1995) Reproducibility and validity of an epidemiologic questionnaire to assess past year physical activity in adolescents. $\mathrm{Am} \mathrm{J}$ Epidemiol 142, 191-201.

24. Rezazadeh A, Rashidkhani B \& Omidvar N (2010) Association of major dietary patterns with socioeconomic and lifestyle factors of adult women living in Tehran, Iran. Nutrition 26, 337-341.

25. Rashidkhani B, Akesson A, Lindblad P et al. (2005) Alcohol consumption and risk of renal cell carcinoma: a prospective study of Swedish women. Int J Cancer 117, 848-853.

26. Hajizadeh B, Rashidkhani B, Rad AH et al. (2010) Dietary patterns and risk of oesophageal squamous cell carcinoma: a case-control study. Public Health Nutr 13, 1107-1112.

27. Baglietto L, Krishnan K, Severi G et al. (2011) Dietary patterns and risk of breast cancer. $B r J$ Cancer 104, 524-531.

28. Brennan SF, Cantwell MM, Cardwell CR et al. (2010) Dietary patterns and breast cancer risk: a systematic review and meta-analysis. Am J Clin Nutr 91, 1294-1302. 
Appendix

\section{Food groupings used in the dietary pattern analyses}

\begin{tabular}{|c|c|}
\hline Food group & Food items \\
\hline Processed meat & Sausages, deli meat, hamburgers \\
\hline Red meat & Beef, lamb \\
\hline Organ meat & Beef liver \\
\hline Fish & Fish \\
\hline Poultry & Chicken \\
\hline Egg & Egg \\
\hline Low-fat dairy products & Skimmed or low-fat milk, low-fat yoghurt, yoghurt drinks \\
\hline High-fat dairy products & $\begin{array}{l}\text { High-fat milk, whole milk, chocolate milk, cream, high-fat yoghurt, cream yoghurt, cream cheese, other } \\
\text { cheese, ice cream }\end{array}$ \\
\hline Tea and coffee & Tea, coffee \\
\hline Fruits & $\begin{array}{l}\text { Pears, apricots, cherries, apples, raisins or grapes, bananas, cantaloupes, watermelons, oranges, } \\
\text { grapefruit, kiwis, strawberries, peaches, nectarines, tangerines, mulberries, plums, persimmons, } \\
\text { pomegranates, lemons, pineapples, fresh figs, fruit juices, dried figs, dried dates, dried mulberries, other } \\
\text { dried fruit }\end{array}$ \\
\hline Industrial juice & Industrial fruit juice \\
\hline Vegetables & $\begin{array}{l}\text { Cabbage, cauliflower, Brussels sprouts, kale, carrots, tomatoes, green leafy vegetables, spinach, lettuce, } \\
\text { cucumber, mixed vegetables, aubergines, celery, green peas, green beans, green pepper, turnip, corn, } \\
\text { squash, mushrooms, onions, garlic, potatoes }\end{array}$ \\
\hline Soya & Soya \\
\hline Legumes & Beans, peas, lima beans, broad beans, lentils \\
\hline Fried potatoes and chips & Fried potatoes and potato chips \\
\hline Whole grains & $\begin{array}{l}\text { Iranian breads (barbari, sangak, taftun, lavash), baguette bread, barley bread, popcorn, cornflakes, wheat } \\
\text { germ, bulgur }\end{array}$ \\
\hline Refined grains & Pasta, rice, toasted bread, milled barley, sweet bread, white flour, starch, biscuits \\
\hline Nuts & Peanuts, almonds, pistachios, hazelnuts, roasted seeds, walnuts \\
\hline Sweets and desserts & Chocolates, cookies, cakes, confectioneries \\
\hline Pickles & Pickles \\
\hline Hydrogenated fats & Hydrogenated fats, animal fats, butter, mayonnaise \\
\hline Olive and vegetable oils & Olive oil, vegetable oils, olives \\
\hline Sugars & Sugars, candies, gaz (Iranian confectionery made of sugar, nuts and egg), jam, jelly, honey \\
\hline Condiments & Turmeric, pepper, others \\
\hline Salt & Salt \\
\hline Soft drinks & Soft drinks \\
\hline
\end{tabular}

